\title{
Pressure-dependent scaling scenarios in experiments of spontaneous imbibition
}

\author{
R. Planet, M. Pradas, A. Hernández-Machado, and J. Ortín* \\ Departament d'Estructura i Constituents de la Matèria, Universitat de Barcelona, Avinguda Diagonal 647, E-08028 Barcelona, Spain
}

(Received 2 May 2007; published 16 November 2007)

\begin{abstract}
The scaling properties of the rough liquid-air interface formed in the spontaneous imbibition of a viscous liquid by a model porous medium are found to be very sensitive to the magnitude of the pressure difference applied at the liquid inlet. Interface fluctuations change from obeying intrinsic anomalous scaling at large negative pressure differences, to being super-rough with the same dynamic exponent $z \simeq 3$ at less negative pressure differences, to finally obeying ordinary Family-Vicsek scaling with $z \simeq 2$ at large positive pressure differences. This rich scenario reflects the relative importance on different length scales of capillary and permeability disorder, and the role of surface tension and viscous pressure in damping interface fluctuations.
\end{abstract}

DOI: 10.1103/PhysRevE.76.056312 PACS number(s): 47.56. $+\mathrm{r}$, 47.15.gp, 68.35.Ct, 05.40. $-\mathrm{a}$

\section{INTRODUCTION}

The dynamics of a fluid propagating into a porous medium has received considerable attention in recent years. Understanding the kinetic roughening process associated with the motion of the liquid-air interface has become a fundamental problem of nonequilibrium statistical physics [1]. It has been studied intensively, both theoretically [2-7] and experimentally [8-17], focusing on the particular case of imbibition.

Spontaneous imbibition is a process of fluid transport in a porous medium, in which a fluid that preferentially wets the medium displaces a resident fluid at constant external pressure. In spontaneous imbibition, the liquid-air interface undergoes a roughening transition under the effect of several competing forces acting on different length scales: while capillary forces tend to destabilize the interface on small length scales, viscous pressure and surface tension tend to stabilize it on large length scales. The result is that the system is spontaneously driven toward a statistically stationary state, with critical interfacial fluctuations [2]. In contrast to other roughening processes, notably those related to the growth or erosion of solid surfaces, the kinetic roughening of a fluid interface in a porous medium is governed by nonlocal dynamics [3-5].

In this paper, we report on the structure and the dynamics of the liquid-air interface, in a series of experiments on spontaneous imbibition of a very viscous fluid by a model porous medium with controlled quenched disorder. Since air can be assumed to have negligible viscosity, the flow is characterized by the dynamic viscosity $\mu$ of the liquid and the surface tension $\sigma$ of the liquid-air interface. These two magnitudes can be combined in the form of a dimensionless capillary number $\mathrm{Ca}=\mu V / \sigma$, where $V$ is the velocity of the flow.

Our model porous medium is a horizontal Hele-Shaw cell with two different values of gap spacing provided by copper squares, randomly distributed in space. Gap thickness fluctuations give rise to (i) capillary pressure fluctuations at the liquid-air interface, (ii) spatial fluctuations in the permeability $\kappa$ of the porous medium, and (iii) fluctuations arising

\footnotetext{
*ortin@ecm.ub.es
}

from the fact that liquid mass conservation in the threedimensional (3D) cell implies nonconservation in the projected 2D problem. The interplay between these different fluctuation sources, acting on different length scales, gives rise to a very rich behavior [5].

The relative importance of capillary and permeability disorder is characterized by a length scale $\xi_{\kappa} \simeq \sqrt{\kappa} / \mathrm{Ca}$. On length scales much smaller than $\xi_{\kappa}$, roughening is dominated by capillary disorder, while permeability disorder is relevant at larger length scales $[2,5]$. On the other hand, given that spontaneous imbibition is a process with slowing-down dynamics, described by Washburn's law [18], a second length scale $\xi_{\times} \simeq \sqrt{\kappa / \mathrm{Ca}}$ also becomes important. $\xi_{\times}$separates surface tension damping from (time-dependent) viscous pressure damping [3].

Previous experiments on imbibition in the same experimental setup [17] showed that, at large negative pressure differences (extremely low interface velocities), the interfacial fluctuations obey intrinsic anomalous scaling [20], with a dynamic exponent $z \simeq 3$ consistent with a nonlocal dynamics driven by capillary forces. The results presented in this paper show that the scaling properties of the interface change significantly with the magnitude of the pressure difference at the liquid inlet, reflecting changes in the relative importance of the different competing forces that come into play [6].

The paper is organized in the following way. A brief review of the scaling of rough interfaces is reported in Sec. II. Details of the experimental setup and procedure are given in Sec. III. The experimental results are presented in Sec. IV. They are put into a theoretical framework in Sec. V. Finally, a discussion and conclusions of our work are given in Sec. VI.

\section{SCALING THEORY OF ROUGH SURFACES}

A fluctuating interface $h(x, t)$ is usually described in terms of its scaling properties. For scale-invariant interfaces one expects the existence of a lateral correlation length growing in time in the form $\ell_{c} \simeq t^{1 / z}$ until it reaches the system size $L$, defining then a saturation time $t_{s} \simeq L^{z}$. The usual tool to describe the scaling properties of the interface fluctuations is the interface global width $\left.w(L, t)=\overline{\left\langle[h(x, t)-\bar{h}]^{2}\right.}\right\rangle^{1 / 2}$, which 
increases as $w(L, t) \sim t^{\beta}$ for $t<t_{s}$ and becomes constant $w(L, t) \sim L^{\alpha}$ for $t \geq t_{s}$. Here, $\langle\cdots\rangle$ denotes the average over different noise realizations and the overbar is a spatial average in the $x$ direction. $\alpha, \beta$, and $z$ are the roughness, growth, and dynamical exponent, respectively, and they are related through the scaling relation $\alpha=z \beta$. In the so-called FamilyVicsek scaling scenario [19], this set of scaling exponents is enough to describe the scaling properties of the interface fluctuations. However, when anomalous scaling is present [20-22], the local scales behave in a different manner from the global ones. Therefore one has to compute the interface width at different window sizes $w(\ell, t)=\langle\langle[h(x, t)$ $\left.\left.\left.-\langle h\rangle_{\ell}\right]^{2}\right\rangle_{\ell}\right\rangle^{1 / 2}$, where $\langle\cdots\rangle_{\ell}$ denotes an average over $x$ in windows of size $\ell<L$. For scale-invariant growth, local fluctuations are expected to increase as

$$
w(\ell, t)=t^{\beta} g\left(\ell / t^{1 / z}\right),
$$

with the corresponding scaling function

$$
g(u) \sim \begin{cases}u^{\alpha_{l o c}} & \text { if } u \ll 1, \\ \text { const } & \text { if } u \gg 1,\end{cases}
$$

where $\alpha_{l o c}$ is the local roughness exponent, which characterizes roughness at small scales. One implication of the anomalous scaling is that the local width saturates when the correlation length reaches the system size, i.e., at times $t_{s}$, and not at the local time $t_{\ell} \sim \ell^{z}$ as occurs in the FamilyVicsek scaling. There is an intermediate regime between $t_{\ell}$ and $t_{s}$ where the local width grows as $w(\ell, t) \sim t^{\beta^{*}}$ with $\beta^{*}$ $=\beta-\alpha_{l o c} / z$.

Alternatively, one can calculate the power spectrum of the interface, $S(k, t)=\left\langle\widetilde{h}_{k}(t) \widetilde{h}_{-k}(t)\right\rangle$, where $\widetilde{h}_{k}(t)$ is the Fourier transform of the interface height. It is also expected to show scaling:

$$
S(k, t)=k^{-(2 \alpha+1)} s_{A}\left(k t^{1 / z}\right),
$$

where now the scaling function has the general form

$$
s_{A}(u) \sim \begin{cases}u^{2\left(\alpha-\alpha_{s}\right)} & \text { if } u \gg 1, \\ u^{2 \alpha+1} & \text { if } u \ll 1,\end{cases}
$$

and $\alpha_{s}$ is the spectral roughness exponent. Different types of scaling arise from this general scaling function [20]. For $\alpha_{s}$ $<1$ it is always true that $\alpha_{l o c}=\alpha_{s}$ and the Family-Vicseck scaling is recovered whenever $\alpha_{s}=\alpha$. In contrast, the socalled intrinsic anomalous scaling appears when $\alpha_{s} \neq \alpha$. Note that for this kind of anomalous scaling a temporal shift is observed in the power spectrum. On the other hand, for $\alpha_{s}$ $>1$, it always occurs that $\alpha_{l o c}=1$, and the super-rough anomalous scaling is obtained if $\alpha=\alpha_{s}$.

\section{EXPERIMENTAL SETUP}

The model porous medium used in this series of experiments was already described in Ref. [17]. It consists of a Hele-Shaw cell of size $190 \times 500 \mathrm{~mm}^{2}$, made of two glass plates separated by a narrow gap. The gap thickness takes two different values, $b=0.46 \mathrm{~mm}$ and $b-\delta b=0.40 \mathrm{~mm}$. The difference in gap thickness is provided by copper squares of
$1.50 \times 1.50 \mathrm{~mm}^{2}$, randomly distributed over a fiberglass substrate, filling $35 \%$ of the total area [13].

The oil gets into the cell from one of its short sides and the displaced air leaves the cell at the other side. The pressure at the oil inlet is kept constant by using an oil container of selectable height $H$ (measured with respect to the height of the air outlet). This fixes the pressure difference between the end of the cell and the liquid inlet as $\Delta p_{a}=\rho g H$. We use a silicone oil (Rhodorsil $47 \mathrm{~V}$ ) with kinematic viscosity $\nu$ $=50 \mathrm{~mm}^{2} / \mathrm{s}$, density $\rho=998 \mathrm{~kg} / \mathrm{m}^{3}$, and oil-air surface tension $\sigma=20.7 \mathrm{mN} / \mathrm{m}$ at room temperature. Once the liquid gets into the model, the oil-air interface is recorded by two charge-coupled device (CCD) cameras with a spatial resolution of $0.34 \mathrm{~mm} / \mathrm{pixel}$, and stored in a computer.

\section{EXPERIMENTAL RESULTS}

Spontaneous imbibition at extremely low interface velocities (achieved by imposing a large negative pressure difference at the oil inlet) was studied in Ref. [17]. Here we study the effect of increasing the applied pressure difference (and hence the range of interface velocities) on the statistical properties of the imbibition front.

We carried out experiments at five different oil-column heights $H$, corresponding to five different pressure differences. For each value of $H$ we used four different disorder configurations, and performed three runs for each configuration. Figure 1 shows the morphology of the interfaces at different pressure differences. We have verified that all experiments follow Washburn's law [18] $\langle h\rangle=A t^{1 / 2}$, as shown in Fig. 2. The range of velocities reached by the average interface in the different experiments is summarized in Table I.

Unavoidable slight perturbations of the interface at the beginning of the experiment make difficult to characterize the scaling of the interfacial fluctuations at very short times. In order to minimize this effect, we have always considered the subtracted width $W(\ell, t)$, defined as $W(\ell, t)=\left[w^{2}(\ell, t)\right.$ $\left.-w^{2}(\ell, 0)\right]^{1 / 2}$, following standard practice $[23,24]$.

It is also important to remark that the interfaces show a slight bend in the plane of the cell, induced by the preferential advancement of the front at the lateral boundaries, where the two ends of the interface meet the gap spacers. Usually this effect is much smaller than the interface fluctuations. For large positive pressure differences, however, the velocity is so large that interfaces are very smooth, and the subtracted width is dominated by the global bending of the interface. In order to eliminate this artifact, the measurements at large pressure differences have been processed in the following way: a second-order polynomial representing the interface background has been fitted to each interface, and the difference between the measured interface and the calculated background has been used for data analysis.

\section{A. Negative pressure differences}

We present here our results for spontaneous imbibition at negative pressure differences. In this case, capillary forces push the interface forward, while the applied pressure differ- 


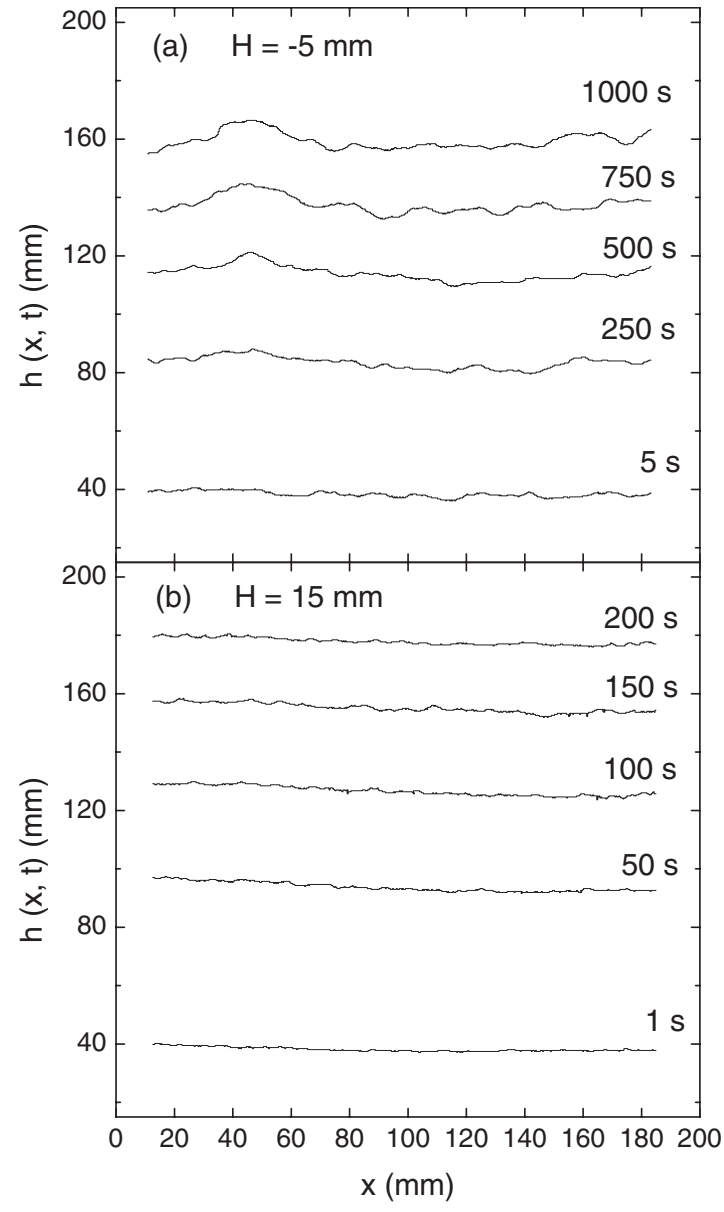

FIG. 1. Sequence of interfaces for experiments with oil-column heights $H=-5$ (top) and $15 \mathrm{~mm}$ (bottom). It can be seen that higher pressure differences lead to smoother interfaces. In each experiment, as time goes on, the interface slows down and gets rougher. The two experiments were carried out with the same disorder configuration.

ence tends to make it recede. At sufficiently large negative pressure differences, this balance of forces makes the interface advance extremely slowly. This capillarity-dominated regime was studied in Ref. [17]. Now, we are interested in studying the behavior of the imbibition front at slightly less

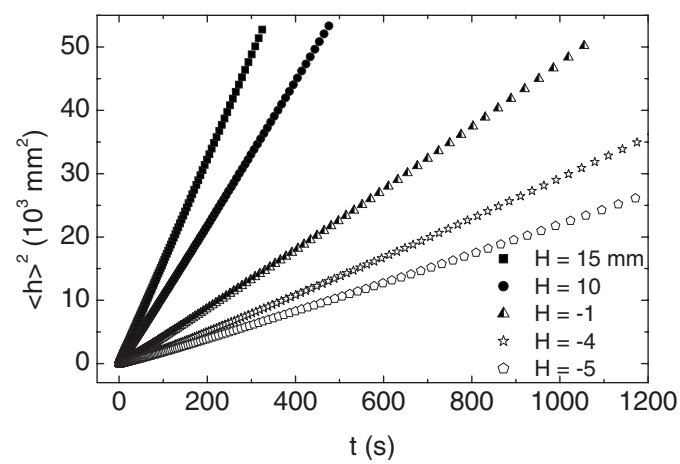

FIG. 2. Squared average interface position $\langle h\rangle$ as a function of time $t$ for the oil-column heights $H$ used in this paper, showing that the interfaces obey Washburn's law $\langle h\rangle=A t^{1 / 2}$.
TABLE I. Summary of velocities (initial and at $\langle h\rangle=350 \mathrm{~mm}$ ), and Washburn's law prefactor, for each of the experiments presented.

\begin{tabular}{cccc}
\hline \hline$H(\mathrm{~mm})$ & $V_{0}(\mathrm{~mm} / \mathrm{s})$ & $V_{\langle h\rangle=350 \mathrm{~mm}}(\mathrm{~mm} / \mathrm{s})$ & $A\left(\mathrm{~mm} / \mathrm{s}^{1 / 2}\right)$ \\
\hline-9 & $0.05 \pm 0.02$ & - & $1.26 \pm 0.15$ \\
-5 & $0.46 \pm 0.11$ & $0.036 \pm 0.003$ & $4.96 \pm 0.10$ \\
-4 & $0.55 \pm 0.12$ & $0.047 \pm 0.003$ & $5.66 \pm 0.10$ \\
-1 & $0.67 \pm 0.20$ & $0.061 \pm 0.004$ & $7.04 \pm 0.10$ \\
10 & $1.36 \pm 0.24$ & $0.17 \pm 0.01$ & $10.68 \pm 0.10$ \\
15 & $1.61 \pm 0.25$ & $0.23 \pm 0.02$ & $12.69 \pm 0.10$ \\
\hline \hline
\end{tabular}

negative pressure differences. The interface in this case moves slightly faster, and the damping effect of viscous pressure on large length scales becomes more relevant. In order to study this regime we present results for two different oilcolumn heights $H=-5$ and $-4 \mathrm{~mm}$.

Figure 3 shows a log-log plot of the interface width as a function of time, for $H=-5 \mathrm{~mm}$, measured with different window sizes and averaged over all the experiments performed in this case. These curves (and also those for $H=$ $-4 \mathrm{~mm}$, not shown) display a power law growth. The power law exponent is different at different length scales. This observation rules out a Family-Vicsek scaling [19], where all length scales follow the same kind of growth. It is important to notice that the interface width does not reach a plateau at long times, meaning that the lateral correlation length grows very slowly and does not reach the whole system size in the course of the experiment.

Figure 4 shows the analysis of the interface fluctuations through the power spectrum. It is possible to see how the larger scales (small $q$ ) get saturated with time: as time increases the power law extends to smaller $q$. The power spectrum at saturation is characterized by a spectral roughness exponent $\alpha_{s}$. Another interesting point to observe is the absence of a temporal shift in the vertical axis for the saturated scales in the figure. This means that $\alpha_{s}=\alpha$, and rules out an intrinsic anomalous scaling scenario, making the present results essentially different from the ones obtained by Soriano et al. [17] for $H=-9 \mathrm{~mm}$. The lack of a temporal shift in the power spectrum (Fig. 4) and the fact that $\alpha_{s}>1$ imply a super-rough scaling scenario.

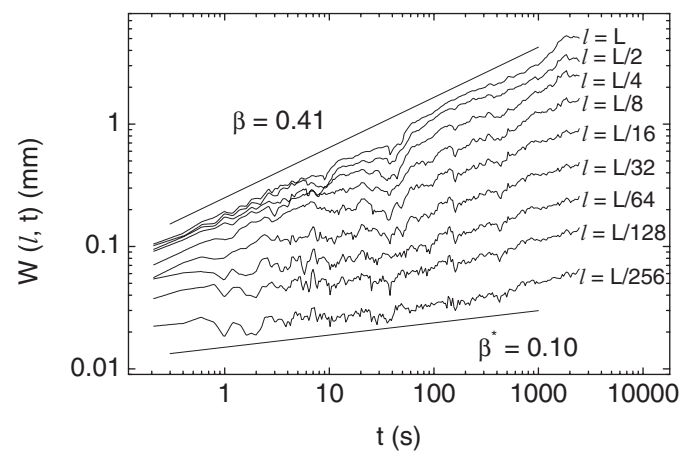

FIG. 3. Log-log plot of the interfacial width as a function of time for different window sizes. The straight lines are linear fits in the scaling region. $H=-5 \mathrm{~mm}$. 


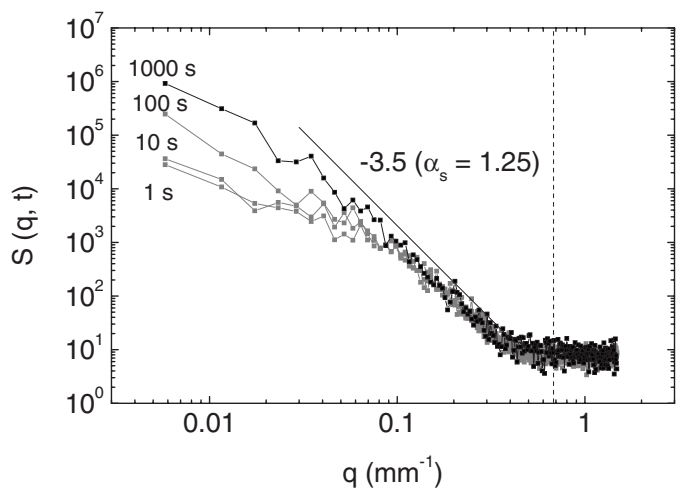

FIG. 4. Power spectrum of the interfacial fluctuations, for $H=$ $-5 \mathrm{~mm}$. The power law provides the value of the spectral roughness exponent $\alpha_{s}$. The vertical dashed line indicates the value $q$ $=0.67 \mathrm{~mm}^{-1}$ associated with the lateral size of the unit's copper obstacle.

With this information and the exponents obtained from the power laws of $W(\ell, t)$ and $S(k, t)$, we can compute all the scaling exponents. The values are given in Table II. In the case of $H=-5 \mathrm{~mm}$ we obtain the exponents $\beta=0.41 \pm 0.02$, $\alpha=1.25 \pm 0.15$, and $z=3.0 \pm 0.4$. The exponents $\beta$ and $z$ derived in this way can then be used to verify the scaling assumption, by collapsing the data for $W(\ell, t)$ in the form shown in Fig. 5. This data collapse (and a similar one obtained for the $H=-4 \mathrm{~mm}$ experiments) confirms that this regime is governed by a lateral correlation length growing in time as $t^{1 / z}$ with a dynamic exponent $z \simeq 3$.

Before going into the positive pressure difference regime, it is worth mentioning that we also performed systematic experiments at an intermediate pressure difference (oilcolumn height $H=-1 \mathrm{~mm}$ ). The results obtained cannot be reconciled in the framework of the theory of dynamic scaling. As discussed below, the lack of scaling must be understood as the result of being in a crossover regime.

\section{B. Positive pressure differences}

When the pressure difference at the oil inlet is positive and large, capillary forces and the pressure difference at the interface, together, push the interface forward-at much larger velocities than in the case just discussed (see Table I). The interfaces now are smoother, as seen in Fig. 1(b), and move with no sign of pinning.

We explored this regime for $H=10$ and $15 \mathrm{~mm}$, and found a common scaling scenario.

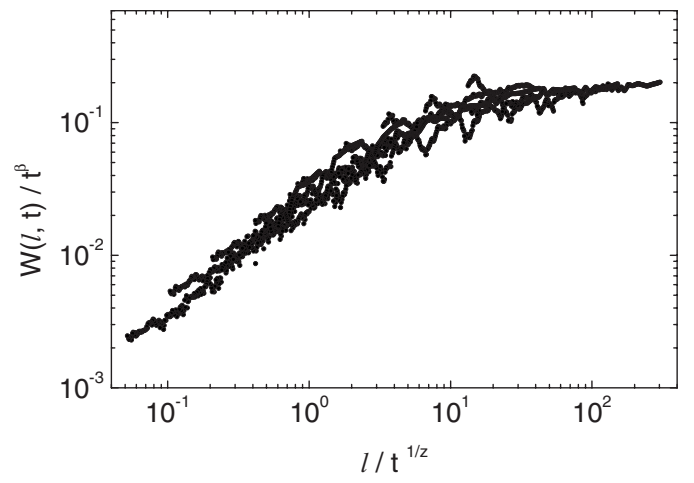

FIG. 5. Data collapse of the interfacial width of Fig. 3 using $\beta=0.41$ and $z=3$. The experiments correspond to $H=-5 \mathrm{~mm}$.

Figure 6 shows a log-log plot of the interfacial width vs time for nine different window sizes, for the experiments with $H=15 \mathrm{~mm}$. The curves display a power law behavior, as in the negative pressure difference regime, but it is important to note that the curve that corresponds to the smallest window size has an exponent very close to zero and thus can also be regarded as having reached saturation very early in the experiment.

Figure 7 is an example of the power spectrum of the interface fluctuations in this regime. As before, the larger length scales become part of the power law at later times, when they reach saturation. The fact that there is no vertical shift of the spectra at different times again in this case rules out the possibility of having intrinsic anomalous scaling, and provides the value of the roughness exponent through the relation $\alpha=\alpha_{s}$.

The complete set of scaling exponents can be derived from the information presented in Figs. 6 and 7. The absence of a temporal shift of the power spectra, as mentioned, allows identifying $\alpha$ with $\alpha_{s}$ and rules out an intrinsic anomalous scaling. Furthermore, the fact that $\alpha<1$ rules out the possibility of having a super-rough scaling. And, in addition, $\beta^{*}$ is very close to 0 (see Table II). This information together points to the conclusion that this regime obeys the usual Family-Vicsek scaling, with the exponents listed in Table II, of which only two are independent. To corroborate this ansatz, it is possible to perform a data collapse of the interfacial width at different time and length scales, using a dynamical exponent $z=2$. The result is shown in Fig. 8 .

\section{THEORETICAL FRAMEWORK}

Fluctuations of gap spacing $b+\delta b$ in the Hele-Shaw cell mainly give rise to two different kinds of disorder acting on

TABLE II. Summary of scaling exponents. See text for details.

\begin{tabular}{ccccccc}
\hline \hline$H(\mathrm{~mm})$ & $\beta$ & $\alpha$ & $\alpha_{\text {loc }}$ & $\beta^{*}$ & $z$ & Type of scaling \\
\hline-9 & $0.64 \pm 0.02$ & $1.94 \pm 0.20$ & $0.94 \pm 0.10$ & $0.33 \pm 0.03$ & $3.0 \pm 0.3$ & Intrinsic anomalous \\
-5 & $0.41 \pm 0.02$ & $1.25 \pm 0.15$ & $0.95 \pm 0.30$ & $0.10 \pm 0.03$ & $3.0 \pm 0.4$ & Super-rough \\
-4 & $0.42 \pm 0.02$ & $1.27 \pm 0.15$ & $0.97 \pm 0.30$ & $0.10 \pm 0.03$ & $3.0 \pm 0.4$ & Super-rough \\
10 & $0.34 \pm 0.02$ & $0.70 \pm 0.15$ & $0.64 \pm 0.20$ & $0.03 \pm 0.02$ & $2.1 \pm 0.4$ & Family-Vicsek \\
15 & $0.36 \pm 0.02$ & $0.70 \pm 0.15$ & $0.59 \pm 0.23$ & $0.06 \pm 0.03$ & $1.9 \pm 0.4$ & Family-Vicsek \\
\hline \hline
\end{tabular}




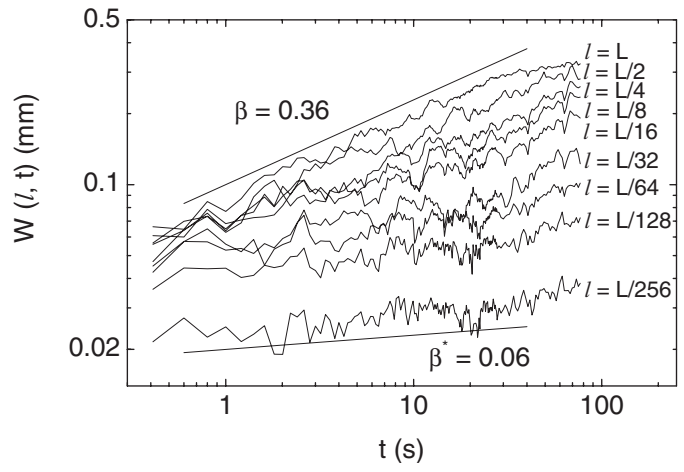

FIG. 6. Log-log plot of the interfacial width as a function of time for different window sizes. The straight lines are linear fits in the scaling region. $H=15 \mathrm{~mm}$.

the interface, capillary disorder and mobility disorder. At low values of the applied pressure difference the capillary disorder $p_{c} \sim 1 /(b+\delta b)$ acts as the main destabilizing term with a global roughness exponent of $\alpha \simeq 1.25$ within the superrough scaling framework, while at higher values of the applied pressure difference the disorder in the permeability $\kappa$ $\sim(b+\delta b)^{2}$ takes an important role in the interface roughening, obtaining then a global roughness exponent of $\alpha \simeq 0.7$ within a Family-Vicsek scaling framework. These regimes are separated by a typical length scale $\xi_{\kappa}[2,5]$.

An estimation of the length scale $\xi_{\kappa}$ can be obtained from Darcy's equation for the interface velocity, $v_{n}=-(\kappa / \mu) \partial_{n} p$. Here $\mu$ is the dynamic viscosity and $p$ the pressure of the viscous liquid, and $n$ is the normal direction at the interface. First, suppose that permeability and pressure can be written as

$$
\begin{aligned}
& \kappa=\kappa_{0}+\delta \kappa, \\
& p=p_{0}+\delta p,
\end{aligned}
$$

where the disorder terms $\delta \kappa$ and $\delta p$ come essentially from the gap variation $\delta b$, and they correspond to permeability and capillary pressure fluctuations, respectively. They can be written as

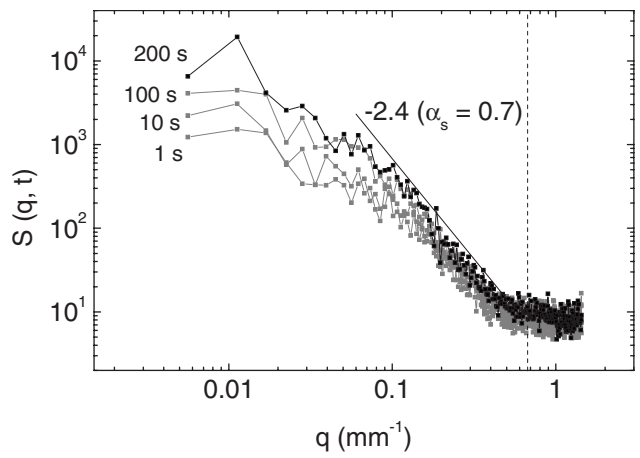

FIG. 7. Log-log plot of the power spectrum at different times, for $H=15 \mathrm{~mm}$. The linear fit in the power law region gives the value of $\alpha_{s}$. The vertical dashed line indicates the value $q$ $=0.67 \mathrm{~mm}^{-1}$ associated with the lateral size of the unit's copper obstacle.

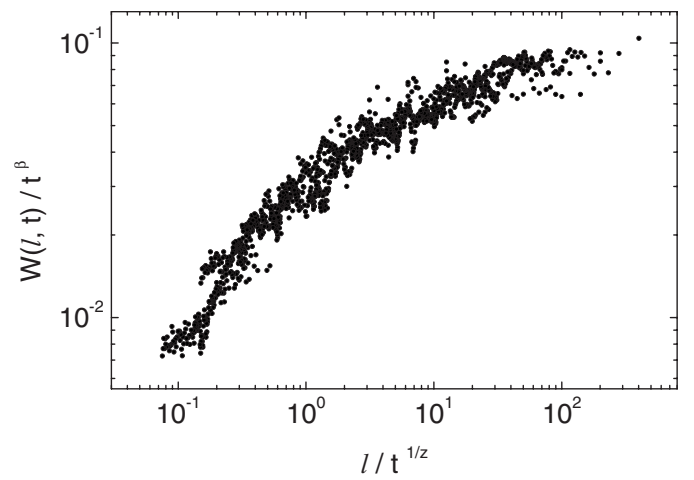

FIG. 8. Data collapse of the interfacial width of Fig. 6 using $\beta=0.36$ and $z=2$. The experiments correspond to $H=15 \mathrm{~mm}$.

$$
\begin{aligned}
& \delta \kappa=2 \kappa_{0} \frac{\delta b}{b}, \\
& \delta p=-\bar{p}_{c} \frac{\delta b}{b},
\end{aligned}
$$

with $\kappa_{0}=b^{2} / 12$ and $\bar{p}_{c}=2 \sigma \cos \theta / b$, where $\sigma$ is the surface tension of the liquid-air interface and $\theta$ is the contact angle. Next, introduce these expressions into Darcy's equation and take linear orders in $\delta b$,

$$
v_{n}=V+2 V \frac{\delta b}{b}+\frac{\kappa_{0} \bar{p}_{c}}{\mu} \partial_{n}\left(\frac{\delta b}{b}\right)
$$

where $V=-\left(\kappa_{0} / \mu\right) \partial_{n} p_{0}$ is the mean velocity of the interface, given by Washburn's law [18] $V(t) \sim t^{-1 / 2}$. Comparing both disorder terms, one obtains the existence of a length scale

$$
\xi_{\kappa} \simeq \frac{\sigma b}{12 \mu V(t)}
$$

separating two regimes. For $\ell_{c}<\xi_{\kappa}$, interface roughness is caused by capillarity (capillary regime). In contrast, for $\ell_{c}$ $>\xi_{\kappa}$, the roughness is produced by the permeability disorder (permeability regime). In order to compare with the experimental correlation length, we use the complete definition of $\xi_{\kappa}=2 \pi \sigma b / 12 \mu V(t)$.

On the other hand, the deterministic linearized equation for the evolution of interface fluctuations in spontaneous imbibition is well known $[2,4,5]$ and can be written, in Fourier space, as

$$
\dot{\tilde{h}}_{q}=-\frac{\sigma \kappa_{0}}{\mu}|q| q^{2} \widetilde{h}_{q}-V(t)|q| \tilde{h}_{q}
$$

This equation shows that, regardless of which is the main disorder, there are two regimes of different modes of controlling the interface roughening [3]. At short scales, the interface fluctuations are controlled by surface tension while, at long scales, the fluctuations are controlled by viscous pressure. These two regimes cross over at the length scale 


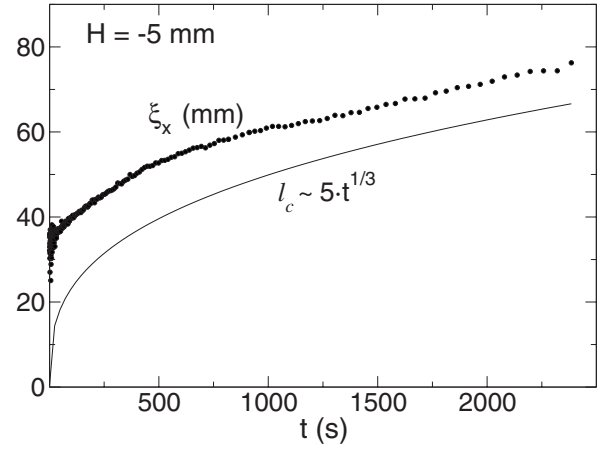

FIG. 9. Crossover length and correlation length versus time for a negative applied pressure difference $(H=-5 \mathrm{~mm})$.

$$
\xi_{\times}=2 \pi\left(\frac{\sigma b^{2}}{12 \mu V(t)}\right)^{1 / 2} .
$$

In order to justify our results, we want to calculate the different length scales present in spontaneous imbibition, that is, $\xi_{\kappa}$ and $\xi_{\times}$, and compare them to the correlation length of the system $\ell_{c}$ for the different regimes studied. It is important to remark that the correlation length $\ell_{c}=B t^{1 / z}$ can only be qualitatively estimated due to the poor precision in calculating the prefactor $B$.

Concerning the scaling properties of Eq. (11), one can obtain the dynamic exponent $z$ at the different regimes separated by $\xi_{\times}$. By imposing the condition that Eq. (11) is scale invariant under the transformation $x \rightarrow b x, h \rightarrow b^{\alpha} h$, and $t$ $\rightarrow b^{z} t$, we obtain that $z=3$ in the surface-tension-dominated regime (taking into account the first term in the right-hand side) and $z=2[25]$ in the viscous-pressure-dominated regime (taking into account the second term in the right-hand side).

\section{A. Negative pressure differences}

For a negative pressure difference $(H=-5 \mathrm{~mm})$, the interface velocity is low enough to guarantee that $\xi_{\kappa} \gg \ell_{c}$ and, therefore, interface fluctuations are described in terms of the capillary disorder. The observed dynamical exponent $z=3$ is the same obtained in Refs. $[6,17]$, and it shows that interface fluctuations are controlled by surface tension [the first term in the right-hand side of Eq. (11)]. We can check it by evaluating the crossover length $\xi_{\times}$, Eq. (12), and comparing it to the correlation length. The result is plotted in Fig. 9 where we observe that $\xi_{\times} \gtrsim \ell_{c}$. The constant $B$ of the correlation length, $\ell_{c}=B t^{1 / z}$, has been estimated from the inflection point of the data collapse in Fig. 5.

When the applied pressure difference is increased up to $H=-1 \mathrm{~mm}$, the initial correlation length can catch up to the crossover length (see Fig. 10). However, a full description of the interface fluctuations cannot be obtained due to the presence of large crossover effects.

\section{B. Positive pressure differences}

In the case of a large pressure difference $(H=15 \mathrm{~mm})$, the interface fluctuations are described by the scaling exponents $z \simeq 1.9$ and $\alpha \simeq 0.7$ within the Family-Vicsek scaling

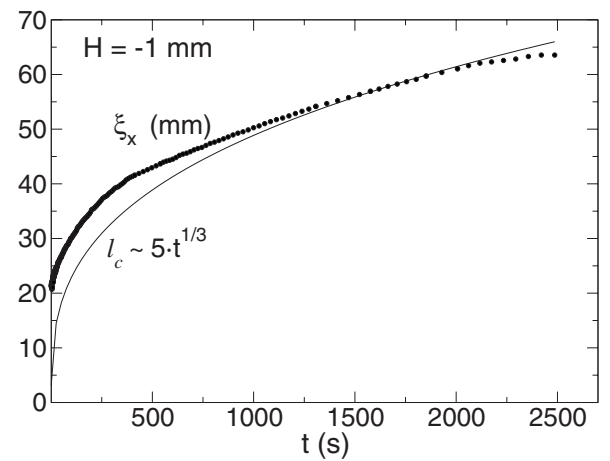

FIG. 10. Crossover length and correlation length versus time for medium applied pressure differences $(H=-1 \mathrm{~mm})$.

description. As before, we want to compare the different length scales. From the saturation time $t_{s} \simeq 90 \mathrm{~s}$ of the global interface width $W(L, t)$ (see Fig. 6), we can calculate the constant $B$ of the correlation length by imposing the condition $\ell_{c}\left(t_{s}\right)=L$. Figure 11 shows that the relation $\ell_{c}$ $\gtrsim \xi_{\kappa} \gg \xi_{\times}$is accomplished, therefore indicating that the permeability noise is more important than, or at least comparable to, the capillary disorder.

\section{DISCUSSION AND CONCLUSIONS}

The results presented in Table II show that the scaling description of the interface fluctuations is greatly affected when the applied pressure is modified. In particular, three different scaling scenarios are observed. At an extremely large negative pressure difference, the interface moves very slowly, under the competing influence of capillary forces that move the interface forward and the external pressure difference that tends to make the interface recede. In this regime, capillary disorder is dominant and there is intrinsic anomalous scaling with a dynamic exponent $z=3$, as reported in Ref. [17].

At larger but still negative pressure differences $(H=-5$ and $-4 \mathrm{~mm}$ ), capillary disorder is still the dominant force of the interface kinetic roughening. Our results show that the main difference from the case of very low velocities is observed at the local scales: intrinsic anomalous scaling has

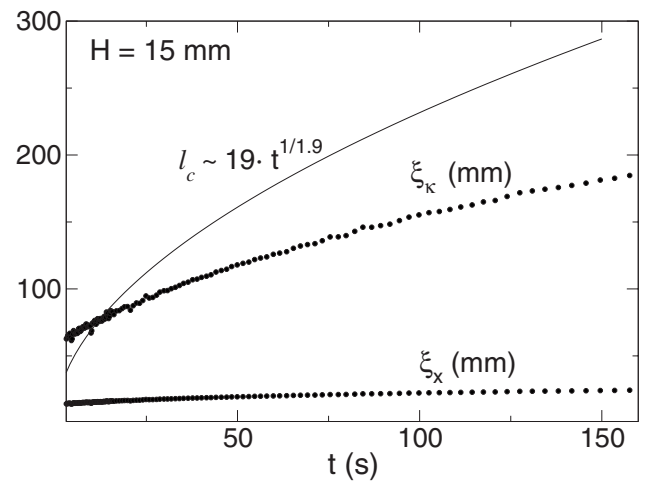

FIG. 11. Different length scales present in spontaneous imbibition for high applied pressure difference $(H=15 \mathrm{~mm})$. 
changed to a super-rough anomalous scaling. As was pointed out in Ref. [6], the presence of intrinsic anomalous scaling in spontaneous imbibition is related to the existence of a high contrast between the different forces acting on the interface: the dichotomic capillary disorder $p_{c} \sim 1 /(b+\delta b)$, and the opposite force due to the applied pressure difference $\Delta p_{a}$ $=\rho g H$. In this sense, increasing the applied pressure difference produces a decrease in the contrast between the two possible values of the resulting force of $p_{c}+\Delta p_{a}$ and, therefore, intrinsic anomalous scaling tends to disappear, giving rise to a super-rough scaling instead. Concerning the global scales, the roughness exponent $\alpha \simeq 1.25$ measured in the experiments is in excellent agreement with the value found numerically in Ref. [3].

When the applied pressure difference is increased up to $H=10$ and $15 \mathrm{~mm}$, the permeability disorder starts to be important in the roughening process $\left(\ell_{c} \gtrsim \xi_{\kappa}\right)$. The situation then becomes clearly different from that in the capillary regime. First, the dynamical exponent changes to $z \simeq 2.0$, reflecting that surface tension is not the mechanism damping the interface fluctuations. Note that the correlation length is much larger than the crossover length (see Fig. 11), indicating that fluctuations are controlled by the second term in the right-hand side of Eq. (11), which is related to the advancement of the front. Taking into account only this term in the interface equation, one can obtain a dynamic exponent $z=2$ by simple power counting, which is very close to the value obtained experimentally. This is a situation never observed in spontaneous imbibition, and in contrast with the numerical results of Refs. $[3,6]$, where only the capillary disorder was considered and a dynamic exponent $z=4$ was obtained. We conclude that the presence of the permeability disorder crucially affects the role of the crossover length scale $\xi_{\times}$. While in the capillary regime it is assumed that the crossover length acts as a correlation length [3], our results in the permeability regime show that this assumption is no longer valid. This has been recently discussed in a wider context of kinetic roughening in Ref. [26].

In conclusion, we have studied the scaling of interfacial fluctuations in the spontaneous imbibition of a viscous fluid by an air-filled porous medium. Our results show that different external pressure differences at the liquid inlet lead to different scaling scenarios. These scenarios reflect the relative importance of capillary and permeability disorder-the two kinds of disorder arising from the quenched disorder of our model porous medium-and the crossover from a surface-tension- to a time-dependent viscous pressuredominated regime.

\section{ACKNOWLEDGMENTS}

We acknowledge enlightening discussions with J. Soriano, and the technical support of A. Comerma and M. Quevedo. R.P. and M.P. acknowledge support from the DGI (MEC, Spain). This work is supported through MEC, Spain, Projects No. FIS2006-03525 and No. FIS2006-12253-C0605, and through Generalitat de Catalunya Project No. SGR2005-00507.
[1] A.-L. Barabási and H. E. Stanley, Fractal Concepts in Surface Growth (Cambridge University Press, Cambridge, England, 1995).

[2] M. Alava, M. Dubé, and M. Rost, Adv. Phys. 53, 83 (2004).

[3] M. Dubé, M. Rost, K. R. Elder, M. Alava, S. Majaniemi, and T. Ala-Nissila, Phys. Rev. Lett. 83, 1628 (1999).

[4] A. Hernández-Machado, J. Soriano, A. M. Lacasta, M. A. Rodríguez, L. Ramírez-Piscina, and J. Ortín, Europhys. Lett. 55, 194 (2001).

[5] E. Pauné and J. Casademunt, Phys. Rev. Lett. 90, 144504 (2003).

[6] M. Pradas and A. Hernández-Machado, Phys. Rev. E 74, 041608 (2006).

[7] M. Rost, L. Laurson, M. Dubé, and M. Alava, Phys. Rev. Lett. 98, 054502 (2007).

[8] M. Sahimi, Flow and Transport in Porous Media and Fractured Rock (John Wiley \& Sons, New York, 1995).

[9] M. A. Rubio, C. A. Edwards, A. Dougherty, and J. P. Gollub, Phys. Rev. Lett. 63, 1685 (1989).

[10] V. K. Horváth, F. Family, and T. Vicsek, J. Phys. A 24, L25 (1991)

[11] S. He, G. L. M. K. S. Kahanda, and P.-Z. Wong, Phys. Rev. Lett. 69, 3731 (1992).

[12] O. Zik, E. Moses, Z. Olami, and I. Webman, Europhys. Lett. 38, 509 (1997).

[13] J. Soriano, J. Ortín, and A. Hernández-Machado, Phys. Rev. E

\section{6, 031603 (2002).}

[14] J. Soriano, J. J. Ramasco, M. A. Rodríguez, A. HernándezMachado, and J. Ortín, Phys. Rev. Lett. 89, 026102 (2002).

[15] D. Geromichalos, F. Mugele, and S. Herminghaus, Phys. Rev. Lett. 89, 104503 (2002).

[16] J. Soriano, J. Ortín, and A. Hernández-Machado, Phys. Rev. E 67, 056308 (2003).

[17] J. Soriano, A. Mercier, R. Planet, A. Hernández-Machado, M. A. Rodríguez, and J. Ortín, Phys. Rev. Lett. 95, 104501 (2005).

[18] E. W. Washburn, Phys. Rev. 17, 273 (1921).

[19] F. Family and T. Vicsek, J. Phys. A 18, L75 (1985).

[20] J. J. Ramasco, J. M. López, and M. A. Rodríguez, Phys. Rev. Lett. 84, 2199 (2000).

[21] J. M. López, M. A. Rodríguez, and R. Cuerno, Phys. Rev. E 56, 3993 (1997).

[22] J. M. López, M. Castro, and R. Gallego, Phys. Rev. Lett. 94, 166103 (2005).

[23] J. H. Jeffries, J.-K. Zuo, and M. M. Craig, Phys. Rev. Lett. 76, 4931 (1996).

[24] G. Tripathy and W. van Saarloos, Phys. Rev. Lett. 85, 3556 (2000).

[25] Note that also Washburn's law $V(t) \sim t^{-1 / 2}$ has to be rescaled.

[26] M. Pradas, J. M. López and A. Hernández-Machado, Phys. Rev. E 76, 010102(R) (2007). 